\title{
PARÁMETROS HEMATOLÓGICOS, CRECIMIENTO Y COMPOSICIÓN CORPORAL DE JUVENILES DE GAMITANA Colossoma macropomum (CUVIER, 1818) CULTIVADOS EN TRES DENSIDADES
}

\author{
Luís Eloy Soberón Minchán', Fred William Chu-Koo y Fernando Alcántara Bocanegra'
}

\section{RESUMEN}

Se evaluaron los posibles efectos de emplear tres densidades de cultivo en jaulas (T1: 10, T2:20 y T3:30 peces $/ \mathrm{m}^{3}$ ) sobre el crecimiento, la composición corporal de grasa y proteina y los parámetros hematológicos de juveniles de gamitana Colossoma macropomum ( $83 \mathrm{~g} \mathrm{y} 16.5 \mathrm{~cm}$ de peso y longitud promedio) alimentados con una dieta extrusada de $25 \%$ de proteína bruta. El ensayo tuvo una duración de 90 días y se ejecutó en el Centro de Investigaciones de Quistococha (CIQ) del IIAP en Loreto, Perú. Se monitoreó el peso y la longitud quincenalmente para evaluar el crecimiento y reajustar las raciones. Los análisis bromatológicos (proteína bruta y extracto etéreo) y hematológicos (glucosa, hemoglobina, hematocrito, número de eritrocitos y leucocitos) se realizaron al inicio y al final del estudio. La calidad de agua (transparencia, dióxido de carbono, alcalinidad, dureza, amonio, nitritos, temperatura, oxígeno disuelto y $\mathrm{pH}$ ) fue monitoreada periódicamente. Se utilizó el ANOVA $(\mathrm{P}<0.05)$ para el análisis de los datos.

No se registró diferencias significativas $(\mathrm{P}>0.05)$ en cuanto al peso final, ganancia de peso, tasa de crecimiento especifico, tasa de crecimiento relativo y factor de condición entre los tratamientos evaluados. Sin embargo, hubo diferencias significativas $(\mathrm{P}<0.05)$ en el indice de conversión alimenticia aparente. Respecto al análisis bromatológico, solamente se registran diferencias significativas $(\mathrm{P}<0.05)$ en el contenido final de proteína bruta. El análisis hematológico solo reportó diferencias significativas $(\mathrm{P}<0.05)$ en los niveles de glucosa. Según los resultados, los peces del T2 $\left(20 \mathrm{peces} / \mathrm{m}^{3}\right)$ tuvieron mejores índices de conversión alimenticia y de contenido corporal de proteína respecto a los peces de los otros tratamientos.

PALABRAS CLAVE: Gamitana, Colossoma macropomum, acuicultura, densidad de cultivo, jaulas, crecimiento, composición corporal, hematología.

\section{HEMATOLOGICAL PARAMETERS, GROWTH AND BODY COMPOSITION OF GAMITANA Colossoma macropomum (CUVIER, 1818) JUVENILES RAISED UNDER THREE STOCKING RATES}

\begin{abstract}
The possible effects of three different stocking rates (T1: 10, T2:20 y T3: $30 \mathrm{fish} / \mathrm{m}^{3}$ ) on juvenile gamitana Colossoma macropomum ( $83 \mathrm{~g} \mathrm{y} 16.5 \mathrm{~cm}$ of average total weight and length) growth, the lipid and protein body composition and hematological parameters were assessed when raised in cages and fed an extruded diet with $25 \%$ of crude protein. The study was conducted during 90 days at the Research Center of Quistococha of IIAP in Loreto, Peru. Fish weight and length was sampled biweekly to evaluate growth. Proximal (protein and lipids) and hematological analysis (glucose, hemoglobin, hematocrit, red blood cells and leukocytes) were carried out both at the beginning and at the end of the trial. Water quality parameters (transparency, carbon dioxide, alkalinity, hardness, ammonia, nitrite, temperature, dissolved oxygen, and $\mathrm{pH})$ were monitored periodically. One-way ANOVA $(\mathrm{P}<0.05)$ was used for data analysis.

No significant differences in final fish body weight, weight gain, daily weight gain, specific growth rate, relative growth rate or condition factor were found $(\mathrm{P}>0.05)$. However, significant differences in feed conversion ratio was found $(\mathrm{P}<0.05)$. Regarding the body composition of the fishes, significant differences were found in final protein content only $(\mathrm{P}<0.05)$. The hematological analysis showed and significant increase in final glucose levels $(\mathrm{P}<0.05)$. According with the results, the fish raised under T2 (20 fish $\left.{ }^{3}\right)$ had a better feed conversion ratio and body protein content than fish cultured under the other two treatments.
\end{abstract}

KEYWORDS: Gamitana, Colossoma macropomum, aquaculture stocking rate, cages, growth, body composition, hematology.

1 Instituto de Investigaciones de la Amazonía Peruana (IIAP). Programa de Ecosistemas Acuáticos (PEA). Centro de Investigaciones de Quistococha (CIa). Carretera Iquitos - Nauta, Km. 4.5, Iquitos, Perú. e-mail: dirpea@iiap.org.pe 


\section{INTRODUCCIÓN}

Uno de los principales factores causante de estrés en piscicultura está asociado a inadecuados niveles de densidad de cultivo y en esas situaciones los peces generalmente presentan bajo crecimiento, hipersecreción de cortisol e hiperglicemia, consideradas como típicas respuestas primarias y secundarias al estrés, respectivamente (Wedemeyer et al. 1990). En tal sentido, las respuestas fisiológicas de los peces en cultivo y sus implicancias en la producción piscícola son temas de sumo interés actual.

La creciente demanda por productos de la acuicultura a nivel mundial a hecho que la intensificación de los sistemas de cultivo vaya en aumento. En ese sentido, se han adoptado sistemas de cultivo intensivo y superintensivo (ej. circuitos cerrados, invernaderos, jaulas flotantes y tanques-red) donde la elevada densidad de peces puede propiciar el desarrollo de factores estresantes, debilitamiento inmunológico y defíciencias nutricionales que comprometen el crecimiento y el estado fisiológico de los peces y por ende los predispone a diferentes tipos de patologías.

Los parámetros hematológicos indican el estado fisiológico de un organismo y se emplean con frecuencia en peces de cultivo para valorar el manejo del cultivo, la efectividad del control de enfermedades infecciosas, posibles desbalances nutricionales, efectos tóxicos, condiciones anóxicas, cambios ambientales y factores generadores de estrés que se pueda presentar en el proceso de crianza (Hrubec et al. 2000; Aydin et al. 2000).

Considerando la importancia de la densidad de cultivo como uno de los factores determinantes en el estado fisiológico de los peces cultivados, el presente estudio está orientado a evaluar los posibles efectos de tres densidades de cultivo, sobre el crecimiento, composición corporal y el estado fisiológico de juveniles de gamitana Colossoma macropomum criados en jaulas sumergidas, mediante la determinación de los principales índices zootécnicos, contenido nutricional de la carne y la cuantificación de los principales parámetros hematológicos de los peces, respectivamente.

\section{MATERIAL Y MÉTODO}

\section{LUGAR DE ESTUDIO, PECES YUNIDADES EXPERIMENTALES}

El estudio se ejecutó en las instalaciones piscícolas del Centro de Investigaciones de Quistococha (CIQ) del Instituto de Investigaciones de la Amazonía Peruana (IIAP). El CIQ está localizado en la margen derecha del Km. 4.5 de la Carretera Iquitos - Nauta.
Políticamente está ubicado en el Distrito de San Juan Bautista, Provincia de Maynas, Departamento de Loreto. Esta zona tiene una temperatura media de 28 ${ }^{\circ} \mathrm{C}$ y una precipitación promedio anual de $2727 \mathrm{~mm}$.

Se utilizó 180 juveniles de gamitana provenientes de estanques de cultivo del IIAP y obtenidos por reproducción inducida vía inducción hormonal realizada en el Laboratorio de Reproducción de Peces del CIQ. Como unidades experimentales se utilizó nueve (9) jaulas experimentales de $1 \times 1 \times 1.2 \mathrm{~m}$ de largo, ancho y alto respectivamente $\left(1.2 \mathrm{~m}^{3}\right)$ constituidas de PVC de 1 pulgada de diámetro, las cuales fueron ubicadas dentro de un estanque piscícola de $5000 \mathrm{~m}^{2}$ (estanque 1B) del CIQ.

\section{DISEÑO EXPERIMENTAL}

Tres densidades de cultivo (T1: 10, T2: 20 y T3: 30 peces $/ \mathrm{m}^{3}$ ) fueron empleadas como tratamientos experimentales y fueron distribuidas al azar por triplicado en las nueve jaulas disponibles. Los efectos de las densidades de cultivo fueron evaluados a través del crecimiento, la composición corporal y los parámetros hematológicos de los peces en cultivo, durante 90 días.

\section{ALIMENTACIÓN DE LOS PECES}

Durante el tiempo que duró el experimento, los peces fueron alimentados con una dieta comercial extrusada con un tenor de $25 \%$ de proteína bruta y 2.6 $\mathrm{Mcal} / \mathrm{Kg}$. de energía digerible, formulada y elaborada en la Planta de Producción de Alimentos Balanceados del IIAP.

Tabla 1. Composición porcentual y proximal de la dieta extrusada utilizada en el cultivo de juveniles de gamitana Colossoma macropomum en jaulas flotantes.

\begin{tabular}{lcc}
\hline \multicolumn{1}{c|}{ INSUMOS } & $\begin{array}{c}\text { VALORES } \\
\text { PORCENTUALES }\end{array}$ \\
\hline Harina de pescado & 1.02 \\
\hline Torta de soya & 42.87 \\
\hline Harina de máz amarillo & 50.00 \\
\hline Sub producto de trigo & 0.70 \\
\hline Premix acuacultura & 0.12 \\
\hline DL - Metionina & 0.04 \\
\hline L - Lisina & 0.06 \\
\hline Cloruro Colina 60\% & 0.10 \\
\hline Carbonato de calcio & 2.50 \\
\hline Fosfato monodicálcico & 2.07 \\
\hline Antioxidante & 0.02 \\
\hline Antimicótico & 0.15 \\
\hline Sal Común & 0.35 \\
\hline Total & 100.00 \\
\hline
\end{tabular}




\begin{tabular}{l|c}
\hline \multicolumn{1}{c|}{$\begin{array}{c}\text { VALOR } \\
\text { NUTRICIONAL }\end{array}$} & $\begin{array}{c}\text { CONTENIDO } \\
(\%)\end{array}$ \\
\hline Materia seca & 88.96 \\
\hline Humedad & 11.04 \\
\hline Proteína & 25.00 \\
\hline Grasa & 3.53 \\
\hline Carbohidratos & 49.15 \\
\hline Fibra & 2.54 \\
\hline Ceniza & 8.58 \\
\hline Energía digest ble Mcal $/ \mathrm{kg}$ & 2.60 \\
\hline
\end{tabular}

La tasa de alimentación empleada fue del $5 \%$ de la biomasa total existente en cada unidad experimental para el primer mes y $3 \%$ desde el segundo mes hasta el final del cultivo, con una frecuencia de alimentación de tres veces al día $(08: 00,12: 00$ y 16:00 h) los siete dias de la semana.

\section{DATOS BIOMÉTRICOS}

Quincenalmente se evaluó el peso y la longitud de todos los peces de cada unidad experimental. Para este proceso se dejó en ayuno a los peces un día antes del muestreo. Momentos antes de la toma de datos se anestesió a los peces con 2-phenoxy-ethanol $(0.02 \%)$ $\mathrm{y}$ posterior a las mediciones, los peces recibieron un baño profiláctico durante tres minutos para luego ser devueltos a sus unidades de origen.

\section{ÍNDICES ZOOTÉCNICOS}

Los índices zootécnicos evaluados fueron: el Índice de Conversión Alimenticia Aparente (ICAA), la Tasa de Crecimiento Especifico (TCE), Tasa de Crecimiento Relativo (TCR), el Factor de Condición (K) y la Sobrevivencia (S).

\section{ANÁLISIS BROMATOLÓGICO}

Se realizó en el Laboratorio de Bromatología y Limnologia del IIAP. Se determinaron los tenores de proteina bruta $(\mathrm{PB})$ y extracto etéreo o grasa (EE) de un pez (completo y triturado) al inicio y al final del experimento. Los análisis bromatológicos fueron realizados siguiendo las recomendaciones de la A.O.A.C modificado (1998).

\section{ANÁLISIS HEMATOLÓGICO}

Se realizó en el Laboratorio de Bromatología y Limnología del ILAP. Para la toma de muestras se anestesió a los peces con 2-phenoxy-ethanol $(0.02 \%)$ y la sangre se extrajo de la arteria caudal con jeringas previamente heparinizadas. Se determinó los valores de glucosa sanguínea, hemoglobina, hematocrito, número de eritrocitos y leucocitos, al inicio $\mathrm{y}$ al final del estudio, según los métodos propuestos por Bush (1982).
Adicionalmente se calculó algunos indices de anemia como:

- Volumen corpuscular medio (VCM)

$$
\operatorname{VCM}(\mathrm{fl})=\begin{gathered}
\text { (porcentaje de hematocrito/ } \\
\text { número de eritrocitos) }
\end{gathered} \times 10
$$

- Hemoglobina corpuscular media(HCM)

$$
\mathrm{HCM}(\mathrm{pg} / \mathrm{cel})=\underset{\text { número de eritrocitos })}{\text { (hemoglobina/ }} \times 10
$$

- Concentración de hemoglobina corpuscular media (CHCM)

$$
\operatorname{CHCM}(\%)=\begin{gathered}
(\text { hemoglobina/porcentaje } \\
\text { de hematocrito })
\end{gathered} \times 10
$$

\section{CALIDADDEL AGUA}

Se realizó el monitoreo periódico de los factores fisico-quimicos del agua, con monitoreos diarios (8, 12 y $16 \mathrm{~h}$ ) de temperatura, oxígeno disuelto y $\mathrm{pH}$, utilizando un medidor multiparámetros YSI modelo MPS 556. Los valores de amonio, nitrito, dureza, alcalinidad, y $\mathrm{CO}_{2}$ fueron monitoreados quincenalmente por las mañanas, utilizando un kit completo para análisis de aguas dulces marca LAMOTTEAQ-2.

\section{ANÁLISIS DELOS DATOS}

Los datos resultantes de las evaluaciones de crecimiento, bromatologia y hematologia fueron procesados en planillas de Excel y analizados a través de ANOVA. Cuando se observó significancia en esta prueba, se aplicó la prueba de comparación de promedios de Tukey (alpha $=0,05$ ) con la ayuda del programa estadístico JMP IN versión 4.0.4. Los valores o resultados expresados en porcentajes fueron transformados por el método del arco seno previo a su análisis en ANOVA. Los resultados son mostrados como el promedio \pm la desviación estándar en cada tratamiento.

\section{RESULTADOS}

\section{CRECIMIENTO DELOS PECES}

Como se indica en la Tabla 2, el peso y la longitud inicial de los peces empleados en el estudio no mostró diferencias significativas ( $P>0.05$ ) lo que significa que el peso y la talla de la población inicial en estudio fueron homogéneas.

Los resultados de la investigación muestran que el crecimiento de los peces fue homogéneo en los tres tratamientos, sin observarse efecto alguno en el peso final, la ganancia de peso corporal ni en la ganancia de peso diario de los peces según ANOVA $(\mathrm{P}>0.05)$, 
\begin{tabular}{l|l} 
FOUIA & PARÁMETROS HEMATOLÓGICOS, CRECIMIENTO Y COMPOSIIIÓN COAPORAL DE JUVENLES
\end{tabular}

\section{ÍNDICES ZOOTÉCNICOS}

En la Tabla 3 se muestran los índices zootécnicos obtenidos en el estudio. No se reportan diferencias significativas $(\mathrm{P}>0.05)$ en TCE, TCR y K por efecto de las densidades. Sin embargo, se observó una probable influencia de los tratamientos experimentales en el ICAA, siendo la conversión alimenticia más apropiada (1.19) obtenida en los peces del $\mathrm{T} 2$ respecto a los T3 y T1 (1.75 y 1.91 respectivamente) según Tukey $(\mathrm{P}<0.05)$. No se registró mortalidad de peces durante la ejecución del estudio.

\section{COMPOSICIÓN CORPORAL}

Se observa una posible influencia de los tratamientos experimentales en la composición corporal de proteína bruta $(74.53 \%$ ), siendo mayor en los peces del T2 respecto a los peces de T3 y T1 (71.47 y $70.49 \%$, respectivamente) según Tukey $(\mathrm{P}<0.05)$. En la Tabla 4 se observa que todos los tratamientos presentaron significativamente un mayor tenor de proteina bruta respecto al valor inicial (67.51) no mostrando diferencias en el contenido corporal de grasas.

Tabla 2. Crecimiento en peso y longitud de juveniles de gamitana $C$. macropomum (promedio \pm desviación estándar) cultivados en tres densidades $\left(10,20\right.$ y 30 peces $\left./ \mathrm{m}^{3}\right)$ durante 90 días (peso corporal inicial: $\mathrm{PCl}$, peso corporal final: PCF, peso corporal ganado: PCG, ganancia de peso diario: GPD, longitud total inicial: LTI, longitud total final: LTF, longitud total ganada: LTG).

\begin{tabular}{l|c|c|c|c}
\hline \multirow{2}{*}{ VARIABLE } & \multicolumn{3}{c|}{ TRATAMIENTO } & \multirow{2}{*}{ PROB. } \\
\cline { 2 - 4 } & $\mathrm{T}_{1}$ & $\mathrm{~T}_{2}$ & $\mathrm{~T}_{3}$ & 0.9117 \\
\hline PCI $(\mathrm{g})$ & $83.06 \pm 0.86 \mathrm{a}$ & $85.53 \pm 8.64 \mathrm{a}$ & $83.50 \pm 9.56 \mathrm{a}$ & 0.4259 \\
PCF $(\mathrm{g})$ & $152.21 \pm 5.84 \mathrm{a}$ & $170.65 \pm 20.58 \mathrm{a}$ & $162.27 \pm 16.50 \mathrm{a}$ & 0.3420 \\
PCG $(\mathrm{g})$ & $69.15 \pm 5.62 \mathrm{a}$ & $81.20 \pm 13.01 \mathrm{a}$ & $78.77 \pm 9.31 \mathrm{a}$ & 0.3570 \\
\hline GPD & $0.77 \pm 0.06 \mathrm{a}$ & $0.90 \pm 0.14 \mathrm{a}$ & $0.87 \pm 0.10 \mathrm{a}$ & 0.6745 \\
LTI $(\mathrm{cm})$ & $16.50 \pm 0.10 \mathrm{a}$ & $16.85 \pm 0.49 \mathrm{a}$ & $16.47 \pm 0.68 \mathrm{a}$ & 0.0889 \\
LTF $(\mathrm{cm})$ & $20.33 \pm 0.06 \mathrm{a}$ & $21.40 \pm 0.56 \mathrm{a}$ & $21.40 \pm 0.70 \mathrm{a}$ & 0.0001 \\
\hline LTG $(\mathrm{cm})$ & $3.83 \pm 0.12 \mathrm{a}$ & $4.55 \pm 0.07 \mathrm{~b}$ & $4.93 \pm 0.06 \mathrm{c}$ & \\
\hline
\end{tabular}

Valores promedio de la misma fila que comparten la misma letra, no muestran diferencias significativas $(P>0.05)$.

Tabla 3. Índices zootécnicos obtenidos en juveniles de gamitana $C$. macropomum (promedio \pm desviación estándar) cultivados en tres densidades $\left(10,20\right.$ y 30 peces/ $\left.\mathrm{m}^{3}\right)$ durante 90 días (índice de conversión alimenticia aparente: ICAA, tasa de crecimiento específico: TCE, tasa de crecimiento relativo: TCR y factor de condición: K).

\begin{tabular}{l|c|c|c|c}
\hline \multirow{2}{*}{ VARIABLE } & \multicolumn{3}{|c|}{ TRATAMIENTO } & \multirow{2}{*}{ PROB. } \\
\cline { 2 - 4 } & $\mathrm{T}_{1}$ & $\mathrm{~T}_{2}$ & $\mathrm{~T}_{3}$ & 0.0327 \\
\cline { 2 - 4 } ICAA & $1.91 \pm 0.10 \mathrm{a}$ & $1.19 \pm 0.38 \mathrm{~b}$ & $1.75 \pm 0.16 \mathrm{ab}$ & 0.3204 \\
$\mathrm{TCE}$ & $0.67 \pm 0.04 \mathrm{a}$ & $0.72 \pm 0.04 \mathrm{a}$ & $0.74 \pm 0.06 \mathrm{a}$ & 0.3120 \\
$\mathrm{~K}$ K & $8.24 \pm 6.27 \mathrm{a}$ & $90.46 \pm 6.94 \mathrm{a}$ & $94.73 \pm 10.05 \mathrm{a}$ & 0.0772 \\
$\mathrm{~S}$ & $1.81 \pm 0.06 \mathrm{a}$ & $1.74 \pm 0.07 \mathrm{a}$ & $1.65 \pm 0.06 \mathrm{a}$ & \\
\hline
\end{tabular}

Valores promedio de la misma fila que comparten la misma letra, no muestran diferencias significativas ( $P>0.05)$.

Tabla 4. Composición corporal (\%) de juveniles de gamitana C. macropomum (promedio \pm desviación estándar) cultivados en tres densidades $\left(10,20\right.$ y 30 peces $\left./ \mathrm{m}^{3}\right)$ durante 90 días (proteína bruta: $\mathrm{PB}$, extracto etéreo: EE).

\begin{tabular}{|c|c|c|c|c|c|}
\hline \multirow{2}{*}{ VARIABLE } & \multicolumn{4}{|c|}{ TRATAMIENTO } & \multirow{2}{*}{ PROB. } \\
\hline & Inicio & $T_{1}$ & $\mathrm{~T}_{2}$ & $T_{3}$ & \\
\hline PB & $67.51 \pm 0.68 a$ & $70.49 \pm 0.65 b$ & $74.53 \pm 0.29 c$ & $71.47 \pm 0.86 b$ & 0.0001 \\
\hline $\mathrm{EE}$ & $14.28 \pm 1.03 a$ & $18.75 \pm 5.89 a$ & $17.94 \pm 6.32 \mathrm{a}$ & $17.20 \pm 3.04 a$ & 0.6712 \\
\hline
\end{tabular}

Valores promedio de la misma fila que comparten la misma letra, no muestran diferencias significativas $(P>0.05)$. 
Tabla 5. Parámetros hematológicos de gamitana C. macropomum (promedio \pm desviación estándar) cultivados en tres densidades $\left(10,20\right.$ y 30 peces $/ \mathrm{m}^{3}$ ) durante 90 días (Glucosa $\mathrm{mg} / \mathrm{dl}$. $=\mathrm{Gluc}$; hemoglobina $\mathrm{g} / \mathrm{dl}$. $=\mathrm{Hb}$; hematocrito $\%=\mathrm{Ht}$; \# de eritrocitos cel/ $\mathrm{mm}^{3} \times 10^{5}=\mathrm{RBC}$; volumen corpuscular medio fl/cel. = VCM; hemoglobina corpuscular media pg/cel. $=\mathrm{HCM}$; concentración de hemoglobina Valores promedio de la misma fila que comparten la misma letra, no muestran diferencias significativas $(P>0.05)$.

\begin{tabular}{|c|c|c|c|c|c|}
\hline \multirow{2}{*}{ VARIABLE } & \multicolumn{4}{|c|}{ TRATAMIENTO } & \multirow{2}{*}{ PROB. } \\
\hline & Inicio & $\mathrm{T}_{1}$ & $\mathrm{~T}_{2}$ & $\mathrm{~T}_{3}$ & \\
\hline Gluc. & $72.67 \pm 7.93 \mathrm{a}$ & $90.75 \pm 8.22 \mathrm{ab}$ & $109.42 \pm 0.12 b$ & $97.35 \pm 23.26 \mathrm{~b}$ & 0.0017 \\
\hline $\mathrm{Hb}$ & $8.81 \pm 1.87 \mathrm{a}$ & $8.74 \pm 0.88 \mathrm{a}$ & $9.23 \pm 1.22 \mathrm{a}$ & $9.12 \pm 0.83 \mathrm{a}$ & 0.8871 \\
\hline $\mathrm{Ht}$ & $29.17 \pm 4.22 a$ & $32.00 \pm 2.83 a$ & $30.67 \pm 5.16 \mathrm{a}$ & $31.83 \pm 4.36 a$ & 0.6376 \\
\hline RBC & $2.28 \pm 0.05 \mathrm{a}$ & $2.14 \pm 0.10 \mathrm{a}$ & $2.30 \pm 0.22 a$ & $2.18 \pm 0.04 a$ & 0.1078 \\
\hline VCM & $128.36 \pm 19.9 a$ & $149.46 \pm 9.86 a$ & $134.31 \pm 24.82 a$ & $146.55 \pm 21.21 \mathrm{a}$ & 0.2356 \\
\hline $\mathrm{HCM}$ & $38.72 \pm 7.32 a$ & $40.85 \pm 3.54 a$ & $40.25 \pm 4.95 \mathrm{a}$ & $41.93 \pm 3.59 a$ & 0.7439 \\
\hline $\mathrm{CHCM}$ & $30.69 \pm 7.69 a$ & $27.29 \pm 0.72 a$ & $31.21 \pm 9.39 a$ & $29.11 \pm 4.70 a$ & 0.7279 \\
\hline Leu & $2.45 \pm 0.25 a$ & $2.26 \pm 0.38 a$ & $2.44 \pm 0.24 a$ & $2.49 \pm 0.10 \mathrm{a}$ & 0.4464 \\
\hline
\end{tabular}

corpuscular media $\%=$ CHCM y $\#$ de leucocitos cel/mm $\mathrm{mm}^{3} \times 10^{3}=$ Leu).

Valores promedio de la misma fila que comparten la misma letra, no muestran diferencias significativas $(P>0.05)$.

\section{PARÁMETROS HEMATOLÓGICOS}

Se observó la influencia de los tratamientos experimentales en el parámetro indicador de estrés, glucosa sanguínea (Gluc) $(109.42 \mathrm{mg} / \mathrm{dl})$, mostrando diferencias significativas $(\mathrm{P}<0.05)$, siendo mayor para el tratamiento dos respecto a los tratamientos tres y uno ( 97.35 y $90.75 \mathrm{mg} / \mathrm{dl}$, respectivamente). A su vez, los tratamientos dos y tres presentaron significativamente un mayor nivel de glucosa sanguinea respecto al valor inicial $(72.67 \mathrm{mg} / \mathrm{dl})$. Sin embargo, el T1 presentó un valor semejante al valor inicial. Los parámetros indicadores de anemia $(\mathrm{Hb}, \mathrm{Ht}, \mathrm{RBC}$, VCM, HCM y CHCM) y el indicador de defensa

\begin{tabular}{|c|c|}
\hline PARÁMETROS & VALOR \\
\hline Temperatura $\left({ }^{\circ} \mathrm{C}\right)$ & $29.68 \pm 0.90$ \\
\hline Oxigeno disuelto (mg/) & $3.24 \pm 0.80$ \\
\hline $\mathrm{pH}(\mathrm{UpH})$ & $5.84 \pm 0.20$ \\
\hline Transparencia $(\mathrm{cm})$ & $42.00 \pm 3.65$ \\
\hline Dióxido de carbono (mg/) & $4.86 \pm 1.21$ \\
\hline Alcalinidad (mg/) & $17.14 \pm 1.07$ \\
\hline Dureza (mg/) & $21.71 \pm 1.80$ \\
\hline Amonio (mg/) & $<0.20$ \\
\hline Nitrito (mgl) & $<0.05$ \\
\hline
\end{tabular}

inmunológica (\# de leucocitos) mostraron valores similares entre tratamientos e inclusive respecto al valor inicial, sin presentar diferencias significativas $(P>0.05)$ tal como se observa en la Tabla 5.

\section{CALIDADDEAGUA}

De acuerdo a los resultados reportados en la Tabla 6, los valores de calidad de agua registrados en la ejecución del estudio se mantuvieron dentro de los rangos normales para el cultivo de gamitana.

\section{DISCUSIÓN}

\section{CRECIMIENTO DE LOS PECES}

El crecimiento observado en el presente estudio fue superior al obtenido por Padilla et al. (1996) quienes cultivaron juveniles de gamitana de $27.4 \mathrm{~g}$ de peso en tanques circulares $(1 \mathrm{pez} / 20 \mathrm{~L})$ durante 85 dias y reportan pesos finales y ganancias de peso máximos de $72.5 \mathrm{~g}$ y $43.7 \mathrm{~g}$, respectivamente. Por otro lado, Chagas \& Val (2003) reportan ganancias de peso similares a las del presente estudio al evaluar el efecto de diferentes niveles de inclusión de vitamina $\mathrm{C}$ como suplemento alimenticio en gamitana. Sin embargo, otras investigaciones reportan mejores niveles de crecimiento en gamitana cultivada en jaulas flotantes. Entre ellos podemos mencionar a Alcántara et al. (2004) quienes consiguieron ganancias de peso superiores a 300 gramos en gamitana y paco (peso inicial de $12 \mathrm{~g}$ ) aunque en ocho meses de cultivo. Por su lado, Calderón \& Baltazar (2006) registraron pesos finales entre 119.43 y $352.53 \mathrm{~g}$ como mínimo y máximo, respectivamente, en un cultivo de gamitana $(8 \mathrm{~g})$ durante 180 dias bajo seis diferentes densidades. 


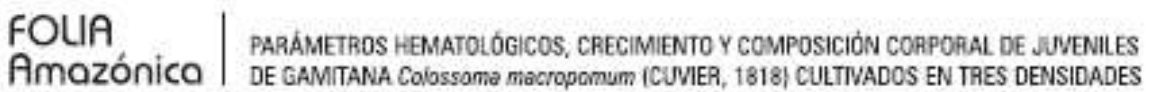

\section{ÍNDICES ZOOTÉCNICOS}

Los ICAA obtenidos en el estudio están dentro del rango normal para el cultivo de gamitana y son evidentemente mejores que los reportados por otros autores como Roubach \& Saint-Paul (1991), quienes alimentando a la misma especie con frutos y semillas de áreas de inundación obtuvieron ICAA mínimos de 1.8 y máximos de 8.9 .

Por su lado, Padilla (2000) obtuvo ICAA de 2.7 y 2.9 al evaluar el contenido proteico-energético de dietas en alevinos de gamitana. En otro estudio, Padilla et al. (2000) obtuvieron ICAA de 3.1 a 3.6 al evaluar la sustitución de la harina de pescado por el ensilado biológico de pescado en raciones para juveniles de gamitana. Del mismo modo, los ICAA del presente estudio obtenidos superan a los obtenidos por Chagas \& Val (2003), quienes obtuvieron entre 1.7 a 3.9 y a Chuquipiondo \& Galdós (2005), quienes obtuvieron ICAA entre 2.1 a 2.2 al evaluar la influencia de la harina de plátano en el crecimiento de alevinos de gamitana, y finalmente a los de Calderón \& Baltazar (2006), quienes obtuvieron ICAA entre 1.3 a 3.2 al evaluar el efecto de seis densidades de cultivo en alevinos de gamitana.

Otros autores reportan ICAA semejantes a los del presente estudio, tales como Gomes et al. (2003) quienes obtuvieron ICAA de 1.27 en alevinos de gamitana cultivada en jaulas flotantes; así también, Chu-Koo \& Chen (2006), quienes registraron ICAA entre 1.8 a 1.9 al evaluar la inclusión tres dietas en el crecimiento, conversión alimenticia y parámetros hematológicos de juveniles de gamitana.

Los niveles de GPD del presente estudio son superiores a los reportados por Padilla et al. (1996) quienes obtuvieron entre 0.44 y $0.52 \mathrm{~g} /$ dia y similares a los obtenidos por Saint-Paul (1984) quien alimentando gamitana con dos raciones conteniendo 27.5 y $42.1 \%$ de $P B$, registró GPD entre 0.8 y $0.9 \mathrm{~g} /$ día. Sin embargo son muchos los autores que reportan valores superiores a los del presente estudio. Por ejemplo, Padilla (2000) reportó valores entre 1.1 y 1.8 g/dia. Padilla et al. (2000) registraron GPD entre 2.24 y $2.65 \mathrm{~g} /$ dia. Del mismo modo, Chu-Koo \& Alván (2006) reportan GPD de 2.35 y $2.78 \mathrm{~g} /$ dia en el cultivo de gamitana y pacotana respectivamente. Finalmente, Chu-Koo \& Chen (2006) reportan GPD entre 2.4 y 2.6 g/día.

Valores de TCE muy superiores a los del presente estudio fueron registrados por Gomes et al. (2003) los cuales variaron entre 5.62 y $9.25 \%$. Del mismo modo, Roubach \& Saint-Paul (1991) reportaron TCE que variaron entre 0.80 y $2.53 \%$.

La tasa de sobrevivencia reportada en el presente estudio pone en evidencia el alto grado de aclimatación de esta especie a nuevos ambientes de cultivo como las jaulas flotantes. Estos resultados son también corroborados por Chagas \& Val (2003). Por el contrario, Moya \& Bances (2001), Rebaza et al. (2002), Rodriguez et al. (2004) y Chuquipiondo \& Galdós (2005) reportaron niveles más bajos de sobrevivencia.

\section{COMPOSICIÓN CORPORAL}

Por naturaleza propia la composición nutricional del pescado es extremadamente variable. En el estado fresco contiene entre 70 a $85 \%$ de humedad, 15 a $24 \%$ de proteina, 0.1 a $22 \%$ de grasa y de 1 a $2 \%$ de material mineral (Ogawa \& Koike, 1987).

Estos porcentajes varian de una especie a otra y también dentro de una misma especie (variación intraespecífica) dependiendo de la época del año, del tipo y cantidad de alimento disponible, de la calidad de la dieta consumida, del estadio de maduración sexual, de la edad, de las condiciones de cultivo y de la parte del cuerpo analizada (Lagler et al. 1977; Castagnolli, 1979; Machado, 1984; Junk, 1985). Asimismo, factores endógenos y/o exógenos pueden influenciar simultáneamente en la composición corporal de los peces (Reinitz \& Hitzel, 1980; Shearer, 1994).

En el presente estudio, el aumento del contenido proteico en la composición corporal de los peces al final del estudio y en especial en los peces del T2 (20 peces $/ \mathrm{m}^{2}$ ) indica que este tratamiento tuvo la densidad de cría más adecuada entre las tres densidades evaluadas, lo que a su vez concuerda con la observación reportada por Moya \& Bances (2001) y Ayllón \& Payahua (2003). El incremento significativo del tenor proteico final respecto al tenor inicial también se puede deber a que los peces como organismos acuáticos gastan menos energía en locomoción y sumado al hecho de tener el alimento a su disposición tres veces al dia reducen notablemente su gasto energético para actividades como desplazamiento y búsqueda de alimento, incidiendo en el uso eficiente de las proteinas para la formación de tejidos.

A esto también se suma que probablemente los carbohidratos de la dieta fueron transformados y almacenados como grasas pues se hizo evidente el incremento de grasa (EE) en los peces al final del estudio en comparación a los niveles iniciales, pero sin llegar a presentar diferencias estadisticas $(P>0.05)$ lo cual concuerda con las experiencias realizadas por otros autores (Cantelmo \& Souza, 1987; Eckman, 1987; Ximenes-Carneiro, 1991 y Padilla, 1996).

\section{HEMATOLOGÍA}

El aumento de la densidad de cultivo es una opción promisoria en piscicultura, la cual combina el máximo 
uso del agua de cultivo asi como el aumento de la producción de peces, siempre teniendo en cuenta que es una fuente potencial de estrés (Barton \& Iwama, 1991; Wedemeyer, 1997) especialmente el estrés crónico, situación en la cual los peces pierden la capacidad homeostática adaptativa (Urbinati \& Carneiro, 2004). Además de ello, las respuestas fisiológicas según indicadores hematológicos, pueden también estar relacionadas a la deficiencia nutricional y metabólica del hierro, causando cuadros de anemia en los peces, lo cual es traducido como retraso en el crecimiento y disminución de la defensa inmunológica, haciéndolos susceptibles a diversas patologias (Roberts, 1981; Shephers \& Bromage, 1988).

En el presente estudio los tratamientos experimentales influyeron en el nivel de glucosa sanguinea (Gluc) registrando diferencias significativas $(\mathrm{P}<0.05)$, siendo los peces del T2 $(109.42 \mathrm{mg} / \mathrm{dl}$.) los que mostraron el mayor nivel de glucosa respecto a los demás tratamientos (T3: 97.35 $\mathrm{mg} / \mathrm{dl}$ y T1: $90.75 \mathrm{mg} / \mathrm{dl}$ ) y muy por encima del nivel de glucosa inicial $(72.67 \mathrm{mg} / \mathrm{dl}$.).

Otros autores también reportan incrementos en el nivel de glucosa respecto al nivel inicial o control en diferentes situaciones en gamitana y especies cercanas. Asi tenemos a Tavares-Dias et al. (2002) que observaron un significativo incremento de glucosa producto de la exposición de $P$. mesopotamicus a concentraciones crecientes de sulfato de cobre $\left(\mathrm{CuSO}_{4}\right)$, un químico usado como medicamento.

La glucosa presente en la sangre de gamitana es una biomolécula principalmente encargada de proporcionarle energia; sin embargo cuando los niveles de glucosa son elevados (hiperglicemia), se puede considerar como un indicador importante en la producción de hormonas relacionadas al estrés en peces en cautiverio, tales como el cortisol y la adrenalina (Gustavenson et al. 1991).

En el presente estudio el alto nivel de glucosa reportado en el T2 no fue suficiente para alterar la fisiología de la gamitana, puesto que la mejor ganancia de peso, índice de conversión alimenticia aparente y tenor proteico fueron registradas en los peces de dicho tratamiento, concordando con valores obtenidos por Tavares-Dias et al. (2002) que obtuvieron valores de glucosa de $74.8,96.5$ y $107.8 \mathrm{mg} / \mathrm{dl}$ en ejemplares de pacú $P$. mesopotamicus tratados con el terapéutico sulfato de cobre $\left(\mathrm{CuSO}_{4}\right)$.

Otras experiencias realizadas con gamitana, demuestran que niveles de glucosa sanguínea superiores a $109.42 \mathrm{mg} / \mathrm{dl}$ (nivel máximo reportado en el presente estudio) no influyen en el normal crecimiento y estado fisiológico de los peces. Por ejemplo, Roubach et al. (2002) reportaron niveles máximos de glucosa entre $110 \mathrm{mg} / \mathrm{dl}$ a $30^{\circ} \mathrm{C}$ y 170 $\mathrm{mg} / \mathrm{dl}$ a $32{ }^{\circ} \mathrm{C}$; en un cultivo de 60 dias de duración realizado a las temperaturas antes mencionadas, registrando ICAA y ganancias de pesos dentro de los rangos normales para el cultivo de gamitana.

Del mismo modo, Affonso et al. (2006) reportan niveles de glucosa entre $80 \mathrm{mg} / \mathrm{dl}$ y $150 \mathrm{mg} / \mathrm{dl}$ al cultivar gamitana durante 150 días bajo dos densidades de cultivo ( 1 y 3 peces $/ \mathrm{m}^{3}$ ), registrando pesos finales de $400 \mathrm{~g}$. Caso contrario sucedió con Castro-Pérez et al. (2002); Chagas et al. (2003); y Chagas et al. (2006), quienes en sus experiencias con gamitana registran niveles de glucosa entre 46.00 $\mathrm{mg} / \mathrm{dly} 74.2 \mathrm{mg} / \mathrm{dl}$.

En cuanto a los parámetros hematológicos indicadores de anemia: hemoglobina ( $\mathrm{Hb})$, hematocrito $(\mathrm{Ht})$; número de eritrocitos (RBC), volumen corpuscular medio (VCM), hemoglobina corpuscular media (HCM) y concentración de hemoglobina corpuscular media $(\mathrm{CHCM})$, observamos que no fueron influenciados por los tratamientos experimentales, mostrando valores semejantes a los del valor inicial $(\mathrm{P}>0.05)$ y son muy semejantes a los obtenidos por autores como Chagas \& Val (2003); Chagas et al. (2006). Sin embargo, los valores de $\mathrm{Hb}, \mathrm{Ht}$ y $\mathrm{RBC}$ obtenidos son ligeramente superiores a los valores de referencia establecidos para esta especie en condiciones de cultivo intensivo en jaulas flotantes (Bastardo \& Ravelo, 2004).

Otros autores como Chagas et al. (2003); Silva et al. (2004); Chu-Koo \& Chen (2006); Affonso et al. (2006), obtuvieron resultados de $\mathrm{Ht}, \mathrm{Hb}$ VCM, HCM y CHCM evidentemente superiores a los del presente estudio. Caso contrario ocurrió con Seibert et al. (2001); Castro-Pérez et al. (2002); Vásquez et al. (2005), quienes reportaron valores de $\mathrm{Hb}$ por debajo del rango de referencia antes mencionado.

El número de leucocitos en la sangre de los peces, indicadores de defensa inmunológica, no presentó diferencia alguna entre tratamientos, mostrando valores semejantes a los niveles iniciales $(\mathrm{P}>0.05)$. $\mathrm{La}$ cantidad de leucocitos sin embargo es evidentemente inferior a los reportados por Tavares-Dias et al. (2002) en gamitana $\left(4600 \mathrm{cel} / \mathrm{mm}^{3}\right)$ y De Andrade et al. (2006) en matrinxã Brycon amazonicus (4200 $\left.\mathrm{cel} / \mathrm{mm}^{3}\right)$.

\section{CALIDADDEAGUA}

Los parámetros de temperatura, $\mathrm{pH}$ y concentración de oxigeno durante los 90 días de cultivo permanecieron dentro de los rangos normales para el cultivo de gamitana $\left(29.68 \pm 0.90^{\circ} \mathrm{C} ; 5.84 \pm\right.$ $0.20 \mathrm{UpH}$ y $3.24 \pm 0.80 \mathrm{mg} / 1 \mathrm{de} \mathrm{O}_{2}$ disuelto respectivamente) lo cual es corroborado por Guerra et 
al. (1996); Padilla (2000) y Rodriguez et al. (2004), quienes reportaron resultados similares.

$\mathrm{La}$ acidez del agua registrada se debe a que un manantial natural (aguajal) fue la fuente principal del suministro de agua al estanque donde se colocaron las jaulas. La transparencia del agua varió entre $42 \pm 3.65$ $\mathrm{cm}$, valor que está dentro del rango normal de transparencia para aguas originarias de la floresta amazónica (aguas negras) según Araújo-Lima \& Goulding (1998) y Alcántara et al. (2002) quienes mencionan que los estanques de piscicultura que presentan una transparencia entre 30 y $60 \mathrm{~cm}$ son los más productivos.

En cuanto al $\mathrm{CO}_{2}$, este parámetro fluctuó entre 4.86 $\pm 1.21 \mathrm{mg} / \mathrm{l}$ no teniendo aparentemente ningún efecto negativo sobre los peces al estar dentro de los valores permisibles según Guerra et al. (1996). La alcalinidad y dureza variaron durante el cultivo entre $17.14 \pm 1.07$ $\mathrm{mg} / 1$ y $21.71 \neq 1.80 \mathrm{mg} / \mathrm{l}$ respectivamente; estos valores están sujetos a la concentración de iones carbonato y bicarbonato para la alcalinidad y de cationes divalentes especialmente del calcio y magnesio para la dureza y están dentro de los rangos normales según Guerra et al. (1996).

Por su lado, los compuestos nitrogenados, producto de la descomposición de material orgánico anduvo por el orden de $0.20 \mathrm{mg} / 1$ y $0.05 \mathrm{mg} / 1$ (de amonio y nitritos, respectivamente) estando dentro de los límites permisibles según Alcántara et al. (2002)

\section{CONCLUSIONES}

A los noventa días de cultivo experimental, los peces ganaron en promedio alrededor de $76.37 \mathrm{~g}$. de peso corporal y el desempeño durante el cultivo fue homogénco.

No se registró mortalidad y los ICAA obtenidos se encuentran dentro de los rangos reportados para el cultivo intensivo de gamitana en jaulas flotantes.

Al final del experimento se observó un incremento del contenido de proteina bruta en la composición corporal de la gamitana.

Los parámetros hematológicos evaluados a excepción del nivel de glucosa sanguínea no fueron influenciados por las densidades de cultivo.

Los parámetros de calidad de agua, permanecieron dentro del rango normal para el cultivo de peces amazónicos.

De las tres densidades evaluadas se observó un desempeño ligeramente más apropiado en los peces del T2 $\left(20\right.$ peces $\left./ \mathrm{m}^{3}\right)$ los cuales presentaron mejores indices de conversión alimenticia y un contenido corporal de proteina mayor respecto a los peces de los otros tratamientos.

\section{AGRADECIMIENTOS}

Al Programa de Ecosistemas Acuáticos (PEA) del Instituto de Investigaciones de la Amazonía Peruana por el financiamiento completo del estudio y la subvención de tesis otorgada para el primer autor del trabajo. Al responsable del Laboratorio de Análisis Clinico de la Cínica Adventista Ana Stahl por la capacitación brindada al primer autor en el área de Hematologia y Bioquimica. Al Blgo. M.Sc. Carlos Chávez Veintemilla y al staff técnico del PEA que colaboraron en la realización del presente estudio.

\section{REFERENCIAS BIBLIOGRÁFICAS}

ABREU J, URBINATI E. Physiological responses of matrinxã (Brycon amazonicus) fed different levels of vitamin $\mathrm{C}$ and submitted to air exposure. Acta Amazónica, 2006;36(4):519-524.

ALCÁNTARA, F, CHÁVEZ C, RODRÍGUEZ L, KOHLER C, KOHLER S, CAMARGO W, COLACEM,TELLOS. G a $m$ i $t$ a $n$ a Colossoma macropomum and Paco Piaractus brachypomus culture in floating cages in Peruvian Amazon. World Aquaculture Society Magazine, 2004; 34 (4): 22-24.

ALCÁNTARA F, KOHLER C, KOHLER, S, CAMARGO W. Cartilla de Acuicultura en la Amazonia. Peruana. IIAP/PD/A CRSP/SIUC/FIAC. 47 pp. 2002.

AFFONSO E, DE OLIVEIRA S, PAULA A, BAPTISTA R, WAICHMAN A, FIM J, ONO E. Caracterização fisiológica de tambaqui, Colossoma macropomum (Characidae) $\mathrm{em}$ duas densidades de estocagem. IV Congreso Iberoamericano Virtual de Acuicultura, 2006. [Consultado el 20 de noviembre del 2007] 8 pp. Disponible en: www.civa2006.org

A.O.A.C. Official Methods of Analysis. International 16th Edition, 4th Revision. 1236 pp. 1998.

ARAÚJO-LIMA C, GOULDING M. Os frutos do tambaqui. Ecologia conservação e cultivo na amazônia. SCM/MCT-CNPq. Brasil.186 pp.1998.

ARBELÁEZ-ROJAS G, MACHADO, INDRUSIAK J. Composição corporal de tambaqui Colossoma macropomum y matrinxã Brycon cephalus, em sistemas de cultivo intensivo, em Igarapé e semiintensivo, em viveiros. Revista Brasileira de Zootecnia, 2002; 38(3):1059-1069.

AYDIN S, GUTELPEAND N, YILDIZ H. Natural and experimental infections of pathology and chemotherapy. Fish Pathology, 2002; 35(3):117-123.

BANZATO DA, KRONKA S. Experimentação 
Agricola. Departamento de Ciências Exatas. Faculdade de Ciências Agrárias e Veterinárias. UNESP. Jaboticabal. SP. 247 pp. 1989.

BARTON B, IWAMA G. Physiological changes in fish from stress in aquaculture with emphasis on the response and effects of corticosteroids. Annual Review of Fish Diseases, 1991; 1(1):3-26.

BASTARDO A, RAVELO C. Control de la anemia en el híbrido Colossoma macropomum x Piaractus brachypomus cultivados mediante el suministro de higado y harina de sangre de ganado vacuno. III Congreso Iberoamericano Virtual de Acuicultura. 2004. [Consultado el 25 de octubre del 2007] 4 pp. Disponible en: www.civa2004.org

BEVERIDGE MCM. Cage aquaculture. Fishing News Books. Oxford. 346 pp. 1996.

BRACK EA. Paradigmas en desarrollo sostenible: Ciencia y tecnología en la Amazonía. CONCYTEC. Lima-Perí. 2002.

BUSH BM. Manual del Laboratorio Veterinario de Análisis Clínicos. Editorial ACRIBIA Zaragoza España. $680 \mathrm{pp} .1982$.

CALDERÓN C, BALTAZAR P. Cultivo de gamitana (Colossoma macropomum) em jaulas flotantes con diferentes densidades. Resúmenes de Exposiciones. 2do Congreso Nacional de Acuicultura. 2006. UNALM-Lima. $7 \mathrm{pp}$.

CANTELMO A, SOUZA JA. Influencia da alimentação em diferentes niveis protéicos para o desenvolvimento inicial do pacu Colossoma mitrei. In: Sintese de trabalhos realizados com espécies do gênero Colossoma. Projeto Aqüicultura. CPTA. Pirassununga. 1987.

CARNEIRO P, URBINATI E. Salt as a stress response mitigator of matrinxã Brycon cephalus (Günther), during transport. Aquaculture Research, 2001; 32(1):297-304.

CASTAGNOLLI N. Fundamentos de nutrição de Peixes. Faculdade de Ciências Agrárias e Veterinárias UNESP. Campus de Jaboticabal. SP. 189 p. 1979 .

CASTAGNOLLI N. Fatores que influenciam a absorção de energia nos peixes. In: Fundamentos de Nutrição de Peixes. São Paulo: Livroceres. 108pp. 1979.

CASTRO-PÉREZ C, SAMPAIO-SOUZA A, DA SILVA R, MOURA L, VAL A. Effects ultraviolet radiation exposure on the swimming performance and hematological parameters of tambaqui Colossoma macropomum. Proceedings of Sixth International Congress on the Biology of Fish. Fish Culture Performance in the Tropics. Manaus, Brazil. 2002; p.133-137.
CHAGAS E; DE GOMES L, MARTINS $\mathrm{H}$, LAURENCO J, ARAÚJO L, DA SILVA, A. Chagas E; De Gomes L, Martins H, Laurenço J, Araújo L, Da Silva, A.

CHAGAS E, LOURENCCO J, DE GOMES L, VALA. Desempenho e estado de saúde de tambaquis cultivados em tanques-rede sob diferentes densidades de estocagem. In: Urbinati E, Cyrino J. Editors. XII Simpósio Brasileiro de Aqüicultura. Aquabio, Jaboticabal, SP. 2003;p. 83-93.

CHAGASE, VALA. Efeito da vitamina $\mathrm{C}$ no ganho de peso e em parâmetros hematológicos de tambaqui. EMBRAPA Amazônia Ocidental INPA. Brasil. Pesq. Agropec. Brás., 2003; 38(3):397-402.

CHAGAS E, DE ARAÚJO L, DA SILVAA, GOMES L, BRANDÃO F. Respostas fisiológicas de tambaqui a banhos terapêuticos com mebendazol. Pesq. agropec. Bras., 2006;41(4):713-716.

CHU-KOOF, CHEN R. Parámetros hematológicos de gamitana (Colossoma macropomum) alimentada com dietas a base de harinas de yuca, plátano y pijuayo. Resúmenes de Exposiciones. 2do Congreso Nacional de Acuicultura. UNALMLima, Peru. 2006.p. 12-13.

CHU-KOO F, ALVÁN J. Resultados preliminares del uso del alimento extrusado en la alimentación de la gamitana Colossoma macropomum y el híbrido pacotana (C. macropomum $\mathrm{x}$ Piaractus brachypomus) en Loreto. Resúmenes de Exposiciones. 2do Congreso Nacional de Acuicultura. UNALM-Lima. 2006. p. 6-7.

CHUQUIPIONDO JML, GALDÓS RAP. Influencia de la harina de plátano, Musa paradisiaca L. en el crecimiento de alevinos de gamitana Colossoma macropomum (Cuvier 1818). Tesis para optar el Título de Biólogo. Facultad de Ciencias Biológicas. Universidad Nacional de la Amazonía Peruana. Iquitos, Perú. 2005.78 pp.

DE ANDRADE J, BRASIL E, ONO E, MATSUMURA T, DE MENEZES G, FERNANDES E, DE OLIVEIRA S, TAVARESDíAS M, AFFONSO E. Influencia de dieta suplementada com vitamina $C$ nas respostas fisiológicas do matrinxã (Brycon amazonicus) após infecção por Aeromonas hydrophila. IV Congreso Iberoamericano Virtual de Acuicultura. 2006. [Consultado el 17 de octubre del 2007] 7 pp. Disponible en: www.civa2006.org

ECKMAN R. Growth and body composition of juvenile Colossoma macropomum CUVIER 1818 (Characidae) feeding artificial diets. Aquaculture, $1987 ; 64: 293-303$. 
GOMES L, BRANDÃO F, CHAGAS E, FERREIRA $\mathrm{M}$, LOURENÇO N. Efeito do volume do tanquerede na produtividade de tambaqui (Colossoma macropomum) durante a recria. Acta Amazónica, 2004;34(1):111-113.

GUERRA H, ALCÁNTARA F, CAMPOS L. Piscicultura amazónica con especies nativas. Tratado de Cooperación Amazónica - TCA. Secretaría Pro Tempore. 169 pp. 1996.

GUSTAVENSONA, WIDORSKI R, WEDEMEYER G. Physiological-response of largemouth bass to angling stress. Trans. American Fisheries Society, 1991; 120(5):29-36.

HRUBEC T, CARDINALE J, SMITH S. Hematology and plasma chemistry reference intervals for cultured tilapia (Oreochromis hibrid). Veterinary Clinical Pathology, 2000; 29(1):7-12.

JUNK JW. Temporary fat storage, an adaptation of some fish species to the water level fluctuations and related environmental changes of the Amazon river. Amazoniana, 1985; 9(3):315-351.

LAGLER K, BARDACH J, MILLER E. Ictiología. México: John Wiley \& Sons. 489 pp. 1977.

MACHADO ZL. Tecnologia de recursos pesqueiros: parâmetros, processos, produtos. Recife: Superintendência de Desenvolvimento da Região Nodeste - Divisão de Recursos Pesqueiros. 277pp. 1984.

MARTINS M, ONAKA E, TAVARES-DÍAS M, BOZZOF, MALHEIROSE. Características hematológicas do híbrido tambacu, 6 e 24 horas após a injeção de substancias irritantes na bexiga natatoria. Revista de Ictiología, 2001; 9(1-2):2531.

MARTINS M, MORAẼS F, FUJIMOTO R, NOMURA D, FENERICK J. Respostas do híbrido tambacu (Piaractus mesopotamicus Holmberg, 1887 macho x Colossoma macropomum Cuvier, 1818 fêmea) a estimulos simples ou consecutivos de captura. Bol. Inst. Pesca, São Paulo, 2002; 28(2): 195-204.

MELO L, IZEL A, RODRIGUES F. Criação de tambaqui (Colossoma macropomum) em viveiros de argila/barragens no Estado do Amazonas. EMBRAPA. Documento 18.30 pp. 2001.

MOYA LC, BANCES KC. Sustitución de la harina de maiz (Zea mays) por la harina de almendro de umari (Poraqueiba sericea) en raciones para alevines de gamitana Colossoma macropomum Pisces, Serrasalmidae. Tesis para optar el Título de Biólogo. Facultad de Ciencias Biológicas. Universidad Nacional de la Amazonía Peruana. Iquitos, Perú. 70 pp. 2001.
OGAWA M, KOIKE J. Manual de pesca. Fortaleza: Associação dos engenheiros de pesca do estado do Ceará. 800 pp. 1987.

PADILLA P, PEREIRA-FILHO M, MORI L. Influencia del ensilado biológico de pescado y pescado cocido en el crecimiento y la composición corporal de alevinos de gamitana, Colossoma macropomum. Folia Amazónica, 1996; 8(2):91103.

PADILLA P, ALCÁNTARA F, GARCÍA J. Sustitución de la harina de pescado por ensilado biológico de pescado en raciones para juveniles de gamitana (Colossoma macropomum). Folia Amazónica, 2000; 10 (1-2):225-240.

PADILLA P. Efecto del contenido proteico y energético de dietas en el crecimiento de alevinos de gamitana (Colossoma macropomum). Folia Amazónica, 2000; 10 (1-2):81-90.

PÉREZ M, DOMITROVIC H. Evaluación de dos parámetros indicadores de estrés en Pacú (Piaractus mesopotamicus). Comunicaciones Científicas y Tecnológicas 2004, Universidad Nacional del Nordeste. Argentina. 3 pp.

REBAZA C, VILLAFANA E, REBAZA M, DEZA S. Influencia de tres densidades de siembra en el crecimiento de Piaractus brachypomus. "paco" en segunda fase de alevinaje en estanques seminaturales. Folia Amazónica, 2002; 13 (12):122-134.

REINITZ G, HITZEL F. Formulation of practical diets for rainbow trout base don desired performance and body composition. Aquaculture, 1980; 19(1):243-252.

ROBERTS R. Patología de los peces. Madrid: Ediciones Mundiprensa. 210 pp. 1981.

RODRIGUES F, CARVALHO L, CAMPOS E, DANTAS L. Densidade de estocagem de juvenil de tambaqui durante a recria em tanques-rede. Pesq. Agropec. Bras., Brasilia. 2004; 39(4):357-362.

ROUBACH R, DE OLIVEIRAA, DE OLIVEIRA N, CHAGASE. Aquaculture of tambaqui and its vitamin $\mathrm{C}$ requirements. Proceedings of Sixth International Congress on the Biology of Fish. Fish Culture Performance in the Tropics. Manaus, Brazil. 2002; 5 pp.

ROUBACHR, SAINT-PAULU. Use of fruits and seeds from Amazonian inundated forests in feeding trials with Colossoma macropomum (Cuvier, 1818) (Pisces, Characidae). Journal of Applied Ichthyology, 1994; 10(1):134-140.

SAINT-PAUL U. Ecological and physiological investigations on Colossoma macropomum, a new 
specie for fish culture in Amazonas. Mems. Asoc. Latinoamerica. Acuicult, 1984; 5(3):501-518.

SAINT-PAUL U. Potential for aquaculture of South American fresh water fishes: a review. Aquaculture, 1986; 54(1):205-240.

SEIBERT C, GUERRA-SHINOARA E, DE CARVALHOE, MARQUESE. Red blood cell parameters and osmotic fragility curve of Colossoma macropomum (Pisces, Osteichthyes, Myleinae) in captivity. Acta Scientiarum, 2001; 23(2):515-520.

SHEARER KD. Factors affecting the proximate composition of cultured fishes with emphasis on salmonids. Aquaculture, 1994; 119(1):63-88.

SHEPHERS J, BROMAGE N. Intensive Fish Farming. Madrid: Professional Books. 126 pp. 1988.

SILVA E, ONO E, AFFONSO E. Caracterização hematological do tambaqui, Colossoma macropomum (Cuvier, 1818), criado em sistema intensive em viveiros escavados com e sem renovação de água. XIV Jornada de iniciação cientifica do PIBIC/CNPq/FAPEAM/INPA. 2004. $2 \mathrm{pp}$.

SILVA E, MENEZES G, DE CARVALHO C, NUNES E, ITUASSÚ D, MARCON J, FIM J, AFFONSO E. 2005. Blood parameters assessment of matrinxã (Brycon cephalus) fed on vitamin supplemented diet. 10pp. Pesq. Agropec. Bras., 2005; 41(1):320-330.

TAVARES-DÍAS M, LATERÇA M, CANELLO S, MAKOTO E, FLORES C, ENGRÁCIA J, RUAS F. Alterações hematológicas e histopatológicas em pacu, Piaractus mesopotamicus Holmberg, 1887. (Osteichthyes, Characidae), tratado com sulfato de cobre (CuSO4). Acta Scientiarum, 2002; 24(2):547-554.

TAVARES-DIAAS $M$, MATAQUEIRO $M$. Características hematológicas, bioquímicas e biométricas de Piaractus mesopotamicus Holmberg, 1887 (Osteichthyes: Characidae) oriundos de cultivo intensivo. Acta Scientiarum, $2004 ; 26(2): 157-162$.
URBINATI E, CARNEIRO P. Práticas de manejo e estresse dos peixes em piscicultura. Em: Cyrino J, Urbinati E, Fracalossi D, Castagnolli N. editors. Tópicos especiais em piscicultura de água doce tropical intensiva. São Paulo: TecArt. 2004.

VAL A, SILVA M, ALMEIDA-VAL V. Hypoxia adaptation in fish of the Amazon: a never-ending task. South African Journal of Zoology, 1998; 33(1):107-114.

VÁSQUEZ R, BASTARDO A, MUNDARAIN I. Ensayo de toxicidad aguda $\mathrm{CL}_{50}-96 \mathrm{~h}$ con acetato de cadmio y parámetros hematológicos en el híbrido cultivado Colossoma macropomum $\mathrm{x}$ Piaractus brachypomus. Zootecnia Tropical, 2005; 23(3):247-257.

WEDEMEYER G, BARTON B, MCLEAY D. Stress and acclimation. In: Schreck CB, Moyle PB. Editors. Methods for fish biology. American Fisheries Society, Bethesda, 1990; p. 451-490.

WEDEMEYER G. Effects of rearing conditions on the health and physiological quality of fish in intensive culture. In: Iwama G, Pickering A, Sumpter J, Schreck C. editors. Fish stress and health in aquaculture. Cambridge: University Press. 1997.

XIMENES-CARNEIRO AR. Elaboração e uso de ensilado biológico de pescado na alimentação de alevinos de tambaqui, Colossoma macropomum (Cuvier, 1818). Dissertacao de Mestrado. Instituto Nacional de Pesquisas da Amazônia/Fundação Universidade de Amazonas. Manaus, Brasil. 81 pp. 1991. 